\section{(D) Check for updates}

Cite this: Food Funct., 2021, 12, 1603

\title{
Polymethoxyflavone purified from Kaempferia parviflora reduces visceral fat in Japanese overweight individuals: a randomised, double-blind, placebo-controlled study $\dagger$
}

\author{
Susumu Yoshino, (D) a Takashi Tagawa, ${ }^{a}$ Riyo Awa, ${ }^{a}$ Jun Ogasawara, ${ }^{a}$ \\ Hiroshige Kuwahara ${ }^{a}$ and Ikuo Fukuhara ${ }^{b}$
}

\begin{abstract}
Visceral fat is a more important factor in obesity-associated disorders in Japanese individuals than in Caucasian individuals. The objective of this randomised, double-blind, placebo-controlled parallel group study, conducted in Japanese overweight adults, was to investigate the effects of polymethoxyflavone purified from Kaempferia parviflora on visceral fat. A total of 80 subjects (aged 20-64 years, $23.0 \leq$ body mass index $<30 \mathrm{~kg} \mathrm{~m}^{-2}$ ) were randomly assigned in 1:1 ratio to either the active (polymethoxyflavone purified from K. parviflora) or placebo group. Over a 12-week period, each subject received two capsules containing polymethoxyflavone purified from $K$. parviflora $(12 \mathrm{mg}$ polymethoxyflavone per day) or placebo. The primary outcome was a reduction in visceral fat area (VFA), while the secondary outcome was a reduction in subcutaneous fat area (SFA) and total fat area (TFA). VFA was measured at 0, 8, and 12 weeks using computed tomography scanning. Results showed that VFA significantly reduced after 12 weeks in the active group and was significantly lower than in the placebo group at 8 and 12 weeks. A significant reduction was observed in SFA and TFA after 8 and 12 weeks in the active group; TFA was significantly lower than that in the placebo group at 8 and 12 weeks. No adverse events associated with the test supplements were observed in either group. Our study shows that administration of polymethoxyflavone purified from $K$. parviflora reduces visceral fat in Japanese overweight adults.
\end{abstract}

Received 11th May 2020 Accepted 1st January 2021

DOI: $10.1039 / \mathrm{dOfo} 01217 \mathrm{c}$

rsc.li/food-function

\section{Introduction}

Obesity increases the risk of metabolic disorders, such as insulin resistance, hyperlipidaemia, and hypertension. ${ }^{1-3}$ Obesity usually develops when the balance between energy intake and energy expenditure is disrupted; it is characterised by an excessive lipid accumulation, resulting in body weight gain. The excessive fat accumulation in adipocytes increases the risk of developing type 2 diabetes associated with insulin resistance. ${ }^{4,5}$ Since the global obesity epidemic is rapidly growing, extensive research is being conducted on drugs and functional foods that can prevent obesity. Studies have reported that some natural products can decrease body fat in humans. ${ }^{6-9}$

Insulin secretion in Japanese is lower than that in Caucasian individuals. ${ }^{10}$ Moreover, visceral fat is an important

\footnotetext{
${ }^{a}$ Research Center, Maruzen Pharmaceuticals, Co., Ltd, Hiroshima 729-3102, Japan. E-mail: S-yoshino@maruzenpcy.co.jp; Tel: +81847525501

${ }^{b}$ Fukuhara Clinic, Hokkaido 061-1351, Japan

$\dagger$ Electronic supplementary information (ESI) available. See DOI: 10.1039/ dofo01217c
}

factor in obesity-associated disorders in Japanese with mild adiposity compared with Caucasian individuals. ${ }^{11}$ Therefore, it is important to reduce visceral fat accumulation in the Japanese population, for which Japan has established diagnostic criteria.

Kaempferia parviflora Wall. ex. Baker (KP) is a perennial plant belonging to the Zingiberaceae family, and is cultivated in Southeast Asia. ${ }^{12} \mathrm{KP}$, also known as Krachai-Dam or black ginger, is popular as a health-promoting herb and has traditionally been used as a folk medicine to reduce blood glucose levels, improve blood flow, and increase vitality. ${ }^{13} \mathrm{KP}$ contains numerous active constituents. These include 25 flavonoids such as various polymethoxyflavone (5,7-dimethoxyflavone; 3,5,7-trimethoxyflavone; 5,7,4'-trimethoxyflavone; 3,5,7, $4^{\prime}$-tetramethoxyflavone; $5,7,3^{\prime}, 4^{\prime}$-tetramethoxyflavone; $3,5,7,3^{\prime}, 4^{\prime}$-pentamethoxyflavone. ${ }^{14-16} \mathrm{KP}$ and polymethoxyflavone have demonstrated many health benefits, such as improvement in blood flow, anti-oxidative, anti-inflammatory, and anti-allergic properties, and amelioration in gastric ulcers. ${ }^{13,16-19}$ We focused on metabolic disorders that are regarded as social problems and previously investigated the efficacy of KP extract (KPE) on obese mice. ${ }^{20}$ Our previous 
study demonstrated that KPE suppresses high-fat diet-induced obesity by increasing energy metabolism. Another study showed that KPE suppresses increase in body weight, reduces body fat accumulation, and alleviates glucose intolerance in obese mice. ${ }^{21}$ Moreover, oral intake of KPE has been shown to increase energy expenditure and fat oxidation in healthy humans. $^{22,23}$ These findings collectively suggest that KPE exerts body fat-lowering effects via increased energy expenditure. Indeed, our previous placebo-controlled, double-blind study in Japanese subjects $\left(24.0 \leq\right.$ body mass index $\left.(\mathrm{BMI})<30 \mathrm{~kg} \mathrm{~m}^{-2}\right)$ showed that compared to the placebo group, consumption of capsules containing KPE for 12 weeks significantly reduced abdominal fat. ${ }^{16}$ The KPE used in the previous study contained $12 \mathrm{mg}$ of polymethoxyflavone. Therefore, we hypothesised that polymethoxyflavone was the major component that exerted the fat-lowering effect. To test this hypothesis, we prepared polymethoxyflavone purified from KP and investigated the effect of continued ingestion of polymethoxyflavone purified from KP for 12 weeks on reduction in abdominal visceral fat in Japanese subjects. In this study, as all subjects enrolled were Japanese, the criteria of the Japan Society for the Study of Obesity were used: BMI of $23->25 \mathrm{~kg} \mathrm{~m}^{-2}$ is defined as "overweight", and BMI of $25->30 \mathrm{~kg} \mathrm{~m}^{-2}$ is defined as "preobese". ${ }^{24}$

\section{Materials and methods}

\section{Study procedures}

This study protocol was approved by the Miyawaki Orthopedics Clinic Institutional Review Board (approval date: 1 July 2019; approval no: 19036, Hokkaido, Japan). The study was conducted by a contract research organisation, New Drug Research Center Inc. (Hokkaido, Japan) from July 2019 to December 2019 at the Fukuhara Clinic (Hokkaido, Japan), in accordance with the Declaration of Helsinki and the Japanese Ethical Guidelines for Medical and Health Research Involving Human Subjects. This study was registered with the UMIN Clinical Trials Registry as UMIN000037453. The purpose, content, procedures, methods, and potential adverse effects of the study were appropriately explained to the subjects. Signed written informed consent was obtained from all subjects before participating in the study.

\section{Subjects}

The subjects were male and female participants aged 20-64 years and had a BMI between $23->30 \mathrm{~kg} \mathrm{~m}^{-2}$. The Japan Society for the Study of Obesity defines "overweight" as having a BMI of $23-<25 \mathrm{~kg} \mathrm{~m}^{-2}$ and "obese level 1 (preobese)" as having a BMI of $25->30 \mathrm{~kg} \mathrm{~m}^{-2} \cdot{ }^{24}$ The subjects were recruited by New Drug Research Center Inc. (Hokkaido, Japan). The exclusion criteria were as follows: history of severe disease (e.g., heart disease, liver disease, kidney disease, or digestive disease); dyslipidaemia; high blood pressure; high blood glucose; use of supplements or functional foods affecting carbohydrate metabolism and lipid metabolism; possible pregnancy, pregnancy or lactation; excessive alcohol intake; difficulty in collecting blood sample; if more than $200 \mathrm{~mL}$ of blood was donated within the last month prior to this study; constipation for more than 5 days; shift workers; travelling for more than 10 days per month; possible allergic symptoms related to the study's test supplements; participation in other clinical trial; or any other reason for ineligibility as determined by the principal investigator.

\section{Preparation of polymethoxyflavone purified from KP}

Dried KP rhizomes were obtained from selected farms in Thailand. The rhizomes were extracted with $60 \%$ (vol/vol) ethanol, and then evaporated in vacuo to obtain the extract. The extract was then treated with synthetic absorbents (DIAIONTM HP20, Mitsubishi Chemical Corporation, Tokyo, Japan) to purify polymethoxyflavone. After treatment, the purified extract was concentrated, and spray dried with $\gamma$-cyclodextrin and dextrin to obtain $8-12 \%$ polymethoxyflavone. The dried powder (polymethoxyflavone purified from KP) was obtained through strict in-process manufacture control and controlled by detailed analysis indicating its phytochemical and nutritional compositions, microbiological status, and heavy metal content. This powder (polymethoxyflavone purified from KP) with standardised polymethoxyflavone $(8-12 \%)$ was found to contain the following six compounds, as identified by high-performance liquid chromatography: 5,7dimethoxyflavone; 3,5,7-trimethoxyflavone; 5,7,4'-trimethoxyflavone; 3,5,7,4'-tetramethoxyflavone; 5,7,3', $4^{\prime}$-tetramethoxyflavone; 3,5,7,3',4'-pentamethoxyflavone.

\section{Test supplements}

We prepared two types of capsules: one containing polymethoxyflavone purified from KP (with $6 \mathrm{mg}$ polymethoxyflavone) and the other without polymethoxyflavone (placebo). The active supplement was a gelatin capsule containing polymethoxyflavone purified from $\mathrm{KP}$, crystalline cellulose, corn starch, and calcium stearate, whereas the placebo contained cyclodextrin instead of polymethoxyflavone purified from KP. Table 1 shows the nutritional composition of the test supplements. We ensured that the active and placebo capsules were indistinguishable by colour, size, flavour, and packaging.

\section{Target sample size}

Based on the findings of our previous clinical study, ${ }^{16}$ capsules containing polymethoxyflavone purified from KP (with $12 \mathrm{mg}$ polymethoxyflavone) were confirmed to reduce visceral fat by $-4.3 \mathrm{~cm}^{2} \pm 6 \mathrm{~cm}^{2}$ compared to placebo capsules $\left(-0.8 \mathrm{~cm}^{2} \pm\right.$

Table 1 Nutritional composition of test supplements (per capsule)

\begin{tabular}{lll}
\hline & Active & Placebo \\
\hline Energy (kcal) & 2.1 & 2.1 \\
Protein $(\mathrm{g})$ & 0.1 & 0.1 \\
Fat $(\mathrm{g})$ & 0.0 & 0.0 \\
Carbohydrate $(\mathrm{g})$ & 0.4 & 0.4 \\
Polymethoxyflavone $(\mathrm{mg})$ & 6.0 & 0.0
\end{tabular}


$9 \mathrm{~cm}^{2}$ ). The required number of subjects was 38 per group when the significance level was set at $5 \%$, and the power was set at $80 \%$. The predicted dropout rate in this study was assumed to be $5 \%$. Therefore, we estimated the number of subjects required per group to be 40 .

\section{Study design}

A randomised, double-blind, placebo-controlled study was conducted for 12 weeks. Subjects were screened for eligibility over three weeks preceding the test supplement ingestion, followed by visits at $0,4,8$, and 12 weeks for the examination. For each test, anthropometric and circulatory parameters were measured, blood and urine samples were taken, and interviews were conducted. Computed tomography (CT) scanning was performed during the screening test, and at $0,8,12$ weeks. A lifestyle questionnaire was answered by all subjects only during the screening test.

The primary outcome of the study was reduction in visceral fat area (VFA) after regular intake of the active capsule (polymethoxyflavone purified from KP) for 12 weeks. Secondary outcomes were changes in body weight, BMI, body fat ratio, waist circumference, hip circumference, subcutaneous fat area (SFA), total fat area (TFA), total cholesterol, triglyceride, and blood glucose. Safety outcomes were occurrence of adverse events. Abnormalities in clinical laboratory parameters (anthropometric and circulatory parameters, blood biochemistry parameters, haematological parameters, and urinalysis parameters) were also assessed. When an adverse event occurred, the principal investigator followed up until the disappearance of the symptoms or a trend of recovery from the date when the event had set in (last date of the follow-up was 24 December 2019).

Randomisation was performed using a computer-generated permuted block randomised scheme (block size of 4) by Higashi-Shinjuku Clinic, Medical Corporation Meiseikai (Tokyo, Japan). After stratification by age, sex, and VFA, subjects were randomised in 1:1 to receive either active capsules or placebo. All subjects, investigators, and study staff (except for the allocation controller) were blinded to the group assignment throughout the study. The randomisation code was kept confidential until final assessment.

During the course of the study, all subjects took two capsules daily along with dinner meals. Subjects were instructed to retain their usual lifestyle habits, including regular eating, exercise, sleeping, smoking, and drinking habits. Subjects were instructed not to use any other oral medications, dietary supplements, or functional foods that may affect body fat, carbohydrate metabolism, or lipid metabolism. Subjects were prohibited from drinking alcohol and had to finish their evening meal by 21:00 hours on the day before the visit. On the test day, eating, drinking (except water), and smoking were prohibited until the test was completed.

\section{Measurement of abdominal fat area}

Subjects underwent CT scan of the umbilical position, using a Robusto-Ei scanner (Hitashi Medico, Tokyo, Japan). Based on the analysis of abdominal CT scan, VFA and SFA values were calculated using visceral fat measurement software (Fat Scan ${ }^{\mathrm{TM}}$ Ver.3.0, N2 Systems Inc., Osaka, Japan), according to the method described by Tokunaga et $a .^{25}$ The abdominal TFA was calculated as the sum of the VFA and SFA.

\section{Measurement of anthropometric and circulatory parameters}

During each test, the subject's body weight, body fat ratio, waist circumference, hip circumference, systolic blood pressure (SBP), diastolic blood pressure (DBP), and pulse rate were measured. Measurements of height were done only during the screening test. The BMI values were determined from height and body weight. Measurements of blood pressure and pulse rate were conducted after rest for about 5 minutes, with the subjects in sitting position. The body weight and body fat ratio measurements were performed using a dual frequency bioelectrical impedance analysis device (DC-320, Tanita Co., Tokyo, Japan).

\section{Blood biochemistry, haematological examination, and urinalysis}

The biochemical analysis of the fasting blood samples was performed by measuring the concentrations of total protein (TP), albumin (ALB), total bilirubin (T-BIL), aspartate aminotransferase (AST), alanine aminotransferase (ALT), lactate dehydrogenase (LDH), alkaline phosphatase (ALP), $\gamma$-glutamyl transpeptidase $(\gamma$-GT), total cholesterol (TC), triglycerides (TG), blood glucose, uric acid, blood urea nitrogen, creatinine (CRE), sodium, chloride, potassium, and calcium. For haematological analysis, white blood cell count (WBC), red blood cell count (RBC), haemoglobin $(\mathrm{Hb})$, haematocrit $(\mathrm{Ht})$, and platelet count (PLT) were measured. A commercial clinical laboratory (SRL, Inc., Ltd, Hokkaido, Japan) carried out all blood analyses. Semiquantitative analysis of the fasting urine samples was performed to measure ketone bodies, occult blood reaction, urobilinogen, bilirubin, urinary protein, and urinary sugar. All urinalyses were performed by SRL, Inc., Ltd (Hokkaido, Japan).

\section{Record of diet and physical activity in daily life diary}

Subjects recorded the details of their daily meals, snacks, and beverages (except for water) for three days before each test at $0,4,8$, and 12 weeks. Based on the diary meal records and photographs, a nutritionist calculated each subject's calorie intake and dietary composition (protein, fat, and carbohydrate), using Excel Eiyo-Kun version 8 (Kenpakusha Co., Ltd, Tokyo, Japan). Physical activity was measured as the number of steps with a pedometer (FB-740, Tanita Co., Tokyo, Japan), and recorded in a daily life diary. The subjects also recorded the test supplement ingestion, subjective symptoms, and daily activities (including eating habits, exercise habits, alcohol intake, and drug intake) in the daily diary. Their diaries were submitted to the principal investigator, who then interviewed the subjects about their conditions and symptoms. 


\section{Statistical analysis}

Data were expressed as mean \pm standard error. A mixed model analysis with two factors, one, between-subjects (group), while the other, within-subjects (time), was used for primary and secondary outcomes. Statistical analyses were performed using Microsoft Excel® 2013 (Microsoft Corp., Redmond, WA, USA) and SAS® 9.3 (SAS Institute Inc., Cary, NC, USA), with statistical significance established at a two-tailed alpha level of $p<0.05$.

\section{Results}

\section{Baseline subject characteristics}

Among the 80 randomised subjects, 2 declined to participate in the study, while 1 dropped out due to spondylolisthesis; thus, 77 subjects completed the trial (Fig. 1). All 77 subjects underwent CT scanning at 0,8 , and 12 weeks, and were included in the analysis. Baseline subject characteristics are shown in Table 2. There were no significant differences among the active supplement and placebo groups. The compliance with the test supplement in the active and placebo groups was $99.5 \pm 1.1 \%$ and $99.5 \pm 1.0 \%$, respectively.

\section{Energy intake and physical activity}

The daily average values for parameters associated with calorie intake and physical activities are shown in Table 3. The large variability in the amount of physical activities led to analyses being conducted separately for male and female subjects. Calorie intake was significantly reduced after 4 weeks in the
Table 2 Baseline subject characteristics

\begin{tabular}{lll}
\hline & Active & Placebo \\
\hline$n$ & 38 & 39 \\
M/F (number) & $13 / 25$ & $13 / 26$ \\
Smoker (number) & $4(\mathrm{M}: 2 ; \mathrm{F}: 2)$ & 8 (M: $3 ; \mathrm{F}: 5)$ \\
Age (years) & $46.2 \pm 1.6$ & $46.3 \pm 1.3$ \\
Height (cm) & $161.9 \pm 1.6$ & $161.3 \pm 1.5$ \\
Body weight (kg) & $67.8 \pm 1.1$ & $67.0 \pm 1.4$ \\
BMI (kg m ${ }^{-2}$ ) & $25.8 \pm 0.2$ & $25.7 \pm 0.3$ \\
Body fat ratio (\%) & $33.2 \pm 1.2$ & $33.0 \pm 1.2$ \\
Waist circumference (cm) & $90.6 \pm 0.7$ & $90.1 \pm 0.9$ \\
Hip circumference (cm) & $96.9 \pm 0.7$ & $96.6 \pm 0.8$ \\
Waist to Hip ratio & $0.94 \pm 0.01$ & $0.93 \pm 0.01$ \\
SBP (mm Hg) & $117.3 \pm 2.0$ & $115.7 \pm 2.1$ \\
DBP (mm Hg) & $70.5 \pm 1.6$ & $69.9 \pm 1.5$ \\
Pulse (beats per minute) & $69.3 \pm 1.5$ & $69.8 \pm 1.3$
\end{tabular}

Values are presented as mean \pm standard error. Abbreviations: BMI, body mass index; DBP, diastolic blood pressure; F, female; M, male; SBP, systolic blood pressure.

active supplement group (female subjects). Further, calorie intake and carbohydrate intake were significantly lower at 12 weeks in the active supplement group than the placebo group at 12 weeks in female subjects. Physical activity was significantly reduced at 9-12 weeks in the active supplement group (male subjects).

\section{Abdominal fat area}

Table 4 shows the changes in the abdominal fat values (VFA, SFA, and TFA). Primary outcome VFA was significantly reduced

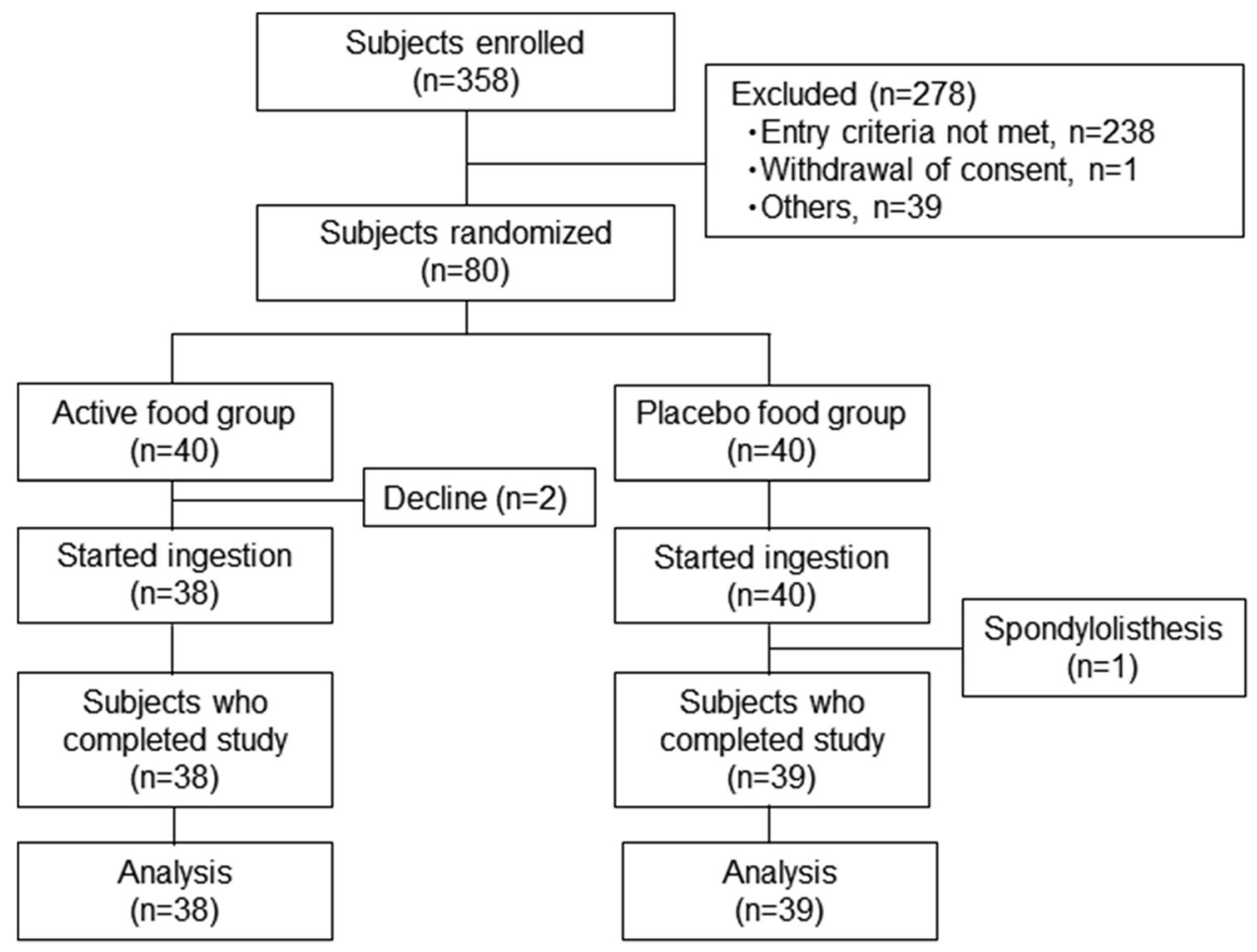

Fig. 1 Flow chart of the present randomized controlled trial. 
Table 3 Energy intake and physical activities

\begin{tabular}{|c|c|c|c|c|c|c|c|}
\hline & Sex & Group & $\mathrm{N}$ & o Week & 4 Weeks & 8 Weeks & 12 Weeks \\
\hline \multirow[t]{4}{*}{ Calorie (kcal) } & \multirow[t]{2}{*}{ Male } & Active & 13 & $1895.9 \pm 109.7$ & $1911.5 \pm 130.8$ & $1902.6 \pm 98.0$ & $1816.5 \pm 82.5$ \\
\hline & & Placebo & 13 & $1911.8 \pm 122.1$ & $1951.0 \pm 130.4$ & $1861.5 \pm 129.4$ & $1880.9 \pm 152.7$ \\
\hline & \multirow[t]{2}{*}{ Female } & Active & 25 & $1844.5 \pm 93.7$ & $1693.5 \pm 88.0^{*}$ & $1845.9 \pm 75.2$ & $1747.3 \pm 71.9$ \\
\hline & & Placebo & 26 & $1698.3 \pm 81.5$ & $1709.0 \pm 57.5$ & $1730.9 \pm 54.1$ & $1806.1 \pm 65.9$ \\
\hline \multirow[t]{4}{*}{ Protein $(\mathrm{g})$} & \multirow[t]{2}{*}{ Male } & Active & 13 & $66.7 \pm 4.2$ & $66.1 \pm 5.7$ & $65.0 \pm 4.9$ & $62.4 \pm 3.5$ \\
\hline & & Placebo & 13 & $62.1 \pm 5.5$ & $63.2 \pm 5.7$ & $56.1 \pm 4.2$ & $59.2 \pm 4.0$ \\
\hline & \multirow[t]{2}{*}{ Female } & Active & 25 & $62.6 \pm 2.7$ & $61.8 \pm 3.7$ & $64.3 \pm 3.2$ & $63.9 \pm 2.4$ \\
\hline & & Placebo & 26 & $61.5 \pm 3.0$ & $61.8 \pm 2.5$ & $64.0 \pm 2.3$ & $68.3 \pm 4.1$ \\
\hline \multirow[t]{4}{*}{ Fat $(g)$} & \multirow[t]{2}{*}{ Male } & Active & 13 & $66.3 \pm 4.9$ & $68.3 \pm 8.6$ & $62.0 \pm 4.2$ & $63.3 \pm 3.8$ \\
\hline & & Placebo & 13 & $58.7 \pm 4.2$ & $67.5 \pm 6.7$ & $56.0 \pm 4.8$ & $56.2 \pm 5.2$ \\
\hline & \multirow[t]{2}{*}{ Female } & Active & 25 & $65.4 \pm 4.8$ & $57.5 \pm 3.6$ & $67.1 \pm 3.8$ & $61.7 \pm 3.8$ \\
\hline & & Placebo & 26 & $57.7 \pm 3.5$ & $59.0 \pm 2.8$ & $60.6 \pm 3.3$ & $59.2 \pm 2.7$ \\
\hline \multirow[t]{5}{*}{ Carbohydrate (g) } & \multirow[t]{2}{*}{ Male } & Active & 13 & $242.4 \pm 14.6$ & $246.8 \pm 13.9$ & $253.3 \pm 17.7$ & $236.3 \pm 10.9$ \\
\hline & & Placebo & 13 & $268.9 \pm 5.2$ & $264.4 \pm 17.9$ & $272.7 \pm 19.9$ & $274.9 \pm 24.8$ \\
\hline & \multirow[t]{3}{*}{ Female } & Active & 25 & $242.8 \pm 14.9$ & $225.9 \pm 14.3$ & $239.4 \pm 12.4$ & $226.0 \pm 11.6 \#$ \\
\hline & & Placebo & 26 & $224.3 \pm 11.3$ & $222.6 \pm 7.2$ & $222.0 \pm 8.7$ & $239.8 \pm 9.0$ \\
\hline & & Group & & 1-4 Weeks & 5-8 Weeks & 9-12 Weeks & 1-12 Weeks \\
\hline \multirow[t]{4}{*}{ Steps (number) } & \multirow[t]{2}{*}{ Male } & Active & 13 & $7931.1 \pm 887.6$ & $7302.5 \pm 854.4$ & $7158.1 \pm 737.2^{*}$ & $7459.5 \pm 805.9$ \\
\hline & & Placebo & 13 & $7564.4 \pm 1152.8$ & $7352.5 \pm 1058.5$ & $7312.5 \pm 999.0$ & $7408.8 \pm 1057.5$ \\
\hline & \multirow[t]{2}{*}{ Female } & Active & 25 & $5845.0 \pm 405.0$ & $5824.1 \pm 407.7$ & $5732.4 \pm 424.0$ & $5802.3 \pm 387.2$ \\
\hline & & Placebo & 26 & $5845.1 \pm 436.7$ & $5538.6 \pm 424.9$ & $5509.0 \pm 412.5$ & $5510.2 \pm 411.0$ \\
\hline
\end{tabular}

Values are presented as mean \pm standard error. \# Significant difference was observed compared with placebo group ( $p<0.05)$. ${ }^{*}$ Significant difference was observed compared with the value at 0 week $(p<0.05)$.

Table 4 Changes in VFA, SFA, and TFA

\begin{tabular}{lllll}
\hline & Group & 0 Week & 8 Weeks & 12 Weeks \\
\hline VFA $\left(\mathrm{cm}^{2}\right) \dagger$ & Active & $85.17 \pm 1.86$ & $82.45 \pm 2.55 \#$ & $81.62 \pm 2.35^{*}, \#$ \\
SFA $\left(\mathrm{cm}^{2}\right) \S$ & Placebo & $83.03 \pm 2.00$ & $84.31 \pm 2.44$ & $84.98 \pm 2.63$ \\
& Active & $235.14 \pm 9.38$ & $224.49 \pm 9.88^{*}$ & $227.55 \pm 9.87^{*}$ \\
TFA $\left(\mathrm{cm}^{2}\right) \dagger, \S$ & Placebo & $233.44 \pm 10.83$ & $229.46 \pm 10.17$ & $230.29 \pm 10.50$ \\
& Active & $320.31 \pm 9.74$ & $306.94 \pm 10.59^{*}, \#$ & $309.17 \pm 10.67^{*}, \#$ \\
& Placebo & $316.47 \pm 11.18$ & $313.77 \pm 11.00$ & $315.27 \pm 11.38$
\end{tabular}

Values are presented as mean \pm standard error; $n=38$ and $n=39$ in the active and placebo groups, respectively. \# Significant differences were observed compared with the placebo group $(p<0.05)$. ${ }^{*}$ Significant differences were observed compared with the values at 0 week $(p<0.05)$. Significant differences were observed at factor of group $(\dagger, p<0.05)$ and of time $(\S, p<0.05)$ using a mixed model. Abbreviations: VFA, visceral fat area; SFA, subcutaneous fat area; TFA, total fat area.

after 12 weeks in the active supplement group, and was significantly lower compared to the placebo at 8 and 12 weeks. Significant difference was observed at the group factor $(p<$ 0.05) using the mixed model.

In the secondary outcome, significant reduction was observed in SFA and TFA after 8 and 12 weeks in the active supplement group, while the TFA was significantly lower compared to the placebo at 8 and 12 weeks. Significant difference was observed at the group $(p<0.05)$ and time $(p<0.05)$ factors in TFA, and at the time factor $(p<0.05)$ in SFA using the mixed model.

\section{Anthropometric values}

The changes observed in anthropometric data before and after treatment are shown in Table 5. A significant increase in the body fat ratio was observed in the placebo group at 8 and 12 weeks compared to that at week 0 . In addition, body fat ratio significantly increased in the active supplement group at 8 weeks compared to that at 0 week. The waist circumference significantly decreased in the active supplement group at 12 weeks compared to that at 0 week, while significantly decreased at 8 weeks in the placebo group. There were no significant differences in waist circumference between both groups throughout the study. There were no significant changes in hip circumference in the placebo group; however, it significantly decreased in the active supplement group at 8 weeks compared to that at 0 week. Further at 12 weeks, a significant difference in the hip circumference between the two groups was observed. No significant changes within the groups or differences between the groups were observed in the waist to hip ratio.

\section{Safety evaluation}

Circulation parameters, blood biochemistry (liver function, lipids and sugar, and kidney function and electrolytes), hae- 
Table 5 Changes in anthropometric parameters

\begin{tabular}{|c|c|c|c|c|c|}
\hline & Group & 0 Week & 4 Weeks & 8 Weeks & 12 Weeks \\
\hline \multirow[t]{2}{*}{ Body weight (kg) } & Active & $67.54 \pm 1.15$ & $67.34 \pm 1.16$ & $67.48 \pm 1.22$ & $67.63 \pm 1.30$ \\
\hline & Placebo & $66.97 \pm 1.36$ & $66.93 \pm 1.38$ & $67.08 \pm 1.35$ & $66.96 \pm 1.36$ \\
\hline \multirow{2}{*}{$\mathrm{BMI}\left(\mathrm{kg} \mathrm{\textrm {m } ^ { - 2 } )}\right.$} & Active & $25.77 \pm 0.27$ & $25.68 \pm 0.26$ & $25.73 \pm 0.28$ & $25.77 \pm 0.29$ \\
\hline & Placebo & $25.66 \pm 0.29$ & $25.66 \pm 0.30$ & $25.72 \pm 0.30$ & $25.66 \pm 0.30$ \\
\hline \multirow[t]{2}{*}{ Body fat $(\%)^{\S}$} & Active & $33.32 \pm 1.21$ & $33.47 \pm 1.21$ & $34.04 \pm 1.21^{*}$ & $33.76 \pm 1.18$ \\
\hline & Placebo & $33.35 \pm 1.23$ & $33.78 \pm 1.18$ & $34.30 \pm 1.19^{*}$ & $33.99 \pm 1.20^{*}$ \\
\hline \multirow[t]{2}{*}{ Waist circumference $(\mathrm{cm}) \S$} & Active & $90.27 \pm 0.60$ & $89.85 \pm 0.68$ & $89.76 \pm 0.81$ & $89.09 \pm 0.82^{*}$ \\
\hline & Placebo & $89.70 \pm 0.92$ & $89.27 \pm 0.89$ & $88.74 \pm 0.90^{*}$ & $89.15 \pm 1.00$ \\
\hline \multirow[t]{2}{*}{ Hip circumference $(\mathrm{cm}) \S$} & Active & $96.86 \pm 0.68$ & $96.50 \pm 0.71$ & $96.04 \pm 0.74^{*}$ & $96.58 \pm 0.80 \#$ \\
\hline & Placebo & $96.34 \pm 0.79$ & $96.55 \pm 0.81$ & $96.09 \pm 0.76$ & $96.74 \pm 0.75$ \\
\hline \multirow[t]{2}{*}{ Waist to Hip ratio } & Active & $0.93 \pm 0.01$ & $0.93 \pm 0.01$ & $0.94 \pm 0.01$ & $0.92 \pm 0.01$ \\
\hline & Placebo & $0.93 \pm 0.01$ & $0.93 \pm 0.01$ & $0.92 \pm 0.01$ & $0.92 \pm 0.01$ \\
\hline
\end{tabular}

Values are presented as mean \pm standard error. $n=38$ and $n=39$ in the active and placebo groups, respectively. ${ }^{\#}$ Significant difference was observed compared with the placebo group $(p<0.05)$. ${ }^{*}$ Significant differences were observed compared with the values at 0 week $(p<0.05)$. Significant differences were observed at factor of time $(\S, p<0.05)$ using a mixed model.

matology, and urinalysis were conducted to assess the safety of polymethoxyflavone. Table 6 shows the results of the circulatory parameters. SBP significantly increased in both the active and placebo groups at 8 and 12 weeks compared to that at 0 week. DBP increased significantly in the placebo group at 8 weeks, while it increased significantly in the active group at 8 and 12 weeks. There were no significant differences in blood pressure parameters (SBP and DBP) between the two groups. There was a significant difference in pulse rate between the two groups after 12 weeks. A significant decrease in pulse rate from the initial value ( 0 week) within a group was observed after 8 and 12 weeks for the active supplement group. Values of all circulatory parameters were within the range of physiological variation.

Tables 7-9 show the results of the blood biochemistry parameters. The levels of LDH and $\gamma$-GT did not significantly change from the initial values ( 0 week) in either within the groups or between the groups. The levels of TP and ALB significantly increased from the values at 0 week in both groups, while no significant differences were observed between the two groups. The levels of T-BIL, AST, and ALT significantly decreased from the values at 0 week in the active supplement group, while no significant differences were observed between the two groups.

The levels of TC and fasting blood glucose significantly increased at 8 weeks and 12 weeks, respectively, from the values at 0 week in both groups, while no significant differences were observed between both groups. No significant changes within or differences between groups were observed in TG.

Serum CRE levels in female subjects significantly decreased from the values at 0 week in the active supplement group, while levels of electrolytes (sodium, chloride, potassium, and calcium) significantly varied from the values at 0 week in both groups. No significant differences were observed between the two groups in kidney function and electrolyte parameters.

The results of haematological parameters are shown in ESI Table $1 . \dagger$ In case of WBC count, no significant changes were observed in both groups for male subjects, whereas significant decrease was observed at 12 weeks from the value at 0 week in the active supplement group for female subjects. In case of RBC count, significant transient increases were observed in both groups for male subjects and in the active supplement group for female subjects. The RBC counts in the active supplement group were significantly lower than those in the placebo group at 0,8 , and 12 weeks in female subjects. In case of $\mathrm{Hb}$, significant increases were observed at 4 weeks from the value at 0 week in the active supplement group for male subjects and at 12 weeks in placebo group for female subjects. The $\mathrm{Hb}$ values in the active supplement group were significantly lower than those in the placebo group at 8 and 12 weeks

Table 6 Changes in circulatory parameters

\begin{tabular}{|c|c|c|c|c|c|c|}
\hline & Reference & Group & 0 Week & 4 Weeks & 8 Weeks & 12 Weeks \\
\hline \multirow[t]{2}{*}{ SBP (mmHg) } & \multirow[t]{2}{*}{$<140$} & Active & $115.0 \pm 1.9$ & $117.7 \pm 1.9$ & $120.3 \pm 2.1^{*}$ & $121.7 \pm 2.2^{*}$ \\
\hline & & Placebo & $112.8 \pm 1.9$ & $117.1 \pm 1.8$ & $119.6 \pm 2.2^{*}$ & $117.7 \pm 2.1^{*}$ \\
\hline \multirow[t]{2}{*}{ DBP (mmHg) } & \multirow[t]{2}{*}{$<90$} & Active & $68.2 \pm 1.5$ & $69.4 \pm 1.6$ & $72.3 \pm 1.6^{*}$ & $72.4 \pm 1.6^{*}$ \\
\hline & & Placebo & $67.4 \pm 1.6$ & $69.2 \pm 1.2$ & $72.8 \pm 1.5^{*}$ & $70.7 \pm 1.7$ \\
\hline \multirow[t]{2}{*}{ Pulse (bpm) } & \multirow[t]{2}{*}{$45-90$} & Active & $67.8 \pm 1.5$ & $67.4 \pm 1.4$ & $65.0 \pm 1.3^{*}$ & $65.4 \pm 1.4^{*}, \#$ \\
\hline & & Placebo & $68.7 \pm 1.5$ & $66.9 \pm 1.4$ & $68.1 \pm 1.3$ & $69.8 \pm 1.4$ \\
\hline
\end{tabular}

Values are presented as mean \pm standard error. $n=38$ and $n=39$ in the active and placebo groups, respectively. \# Significant difference was observed compared with the placebo group $(p<0.05)$. ${ }^{*}$ Significant differences were observed compared with the values at 0 week $(p<0.05)$. Abbreviations: SBP, systolic blood pressure; DBP, diastolic blood pressure. 
Table 7 Changes in blood biochemistry parameters (liver function)

\begin{tabular}{|c|c|c|c|c|c|c|}
\hline & Reference & Group & 0 Week & 4 Weeks & 8 Weeks & 12 Weeks \\
\hline \multirow[t]{2}{*}{$\mathrm{TP}\left(\mathrm{g} \mathrm{dL}^{-1}\right)$} & \multirow[t]{2}{*}{$6.7-8.3$} & A & $7.17 \pm 0.05$ & $7.28 \pm 0.06^{*}$ & $7.33 \pm 0.06^{*}$ & $7.31 \pm 0.06^{*}$ \\
\hline & & $\mathrm{P}$ & $7.20 \pm 0.05$ & $7.31 \pm 0.04^{*}$ & $7.43 \pm 0.05^{*}$ & $7.39 \pm 0.05^{*}$ \\
\hline \multirow[t]{2}{*}{$\operatorname{ALB}\left(\mathrm{g} \mathrm{dL}^{-1}\right)$} & \multirow[t]{2}{*}{$3.8-5.2$} & $\mathrm{~A}$ & $4.28 \pm 0.04$ & $4.30 \pm 0.04$ & $4.37 \pm 0.04^{*}$ & $4.37 \pm 0.05^{*}$ \\
\hline & & $\mathrm{P}$ & $4.32 \pm 0.04$ & $4.34 \pm 0.04$ & $4.44 \pm 0.05^{*}$ & $4.46 \pm 0.04^{*}$ \\
\hline \multirow[t]{2}{*}{ T-BIL $\left(\mathrm{mg} \mathrm{dL}^{-1}\right)$} & \multirow[t]{2}{*}{$0.3-1.2$} & $\mathrm{~A}$ & $0.72 \pm 0.05$ & $0.72 \pm 0.05$ & $0.67 \pm 0.04$ & $0.64 \pm 0.04^{*}$ \\
\hline & & $\mathrm{P}$ & $0.74 \pm 0.05$ & $0.71 \pm 0.04$ & $0.72 \pm 0.05$ & $0.71 \pm 0.04$ \\
\hline \multirow[t]{2}{*}{$\operatorname{AST}\left(\mathrm{U} \mathrm{L}^{-1}\right)$} & \multirow[t]{2}{*}{$10-40$} & $\mathrm{~A}$ & $21.4 \pm 1.0$ & $22.6 \pm 1.4$ & $22.9 \pm 1.1$ & $20.0 \pm 0.9^{*}$ \\
\hline & & $\mathrm{P}$ & $22.2 \pm 2.4$ & $20.2 \pm 0.7$ & $21.8 \pm 0.9$ & $20.4 \pm 1.5$ \\
\hline \multirow[t]{2}{*}{$\operatorname{ALT}\left(\mathrm{U} \mathrm{L}^{-1}\right)$} & \multirow[t]{2}{*}{$5-40$} & A & $25.6 \pm 2.0$ & $28.9 \pm 3.4$ & $26.3 \pm 2.3$ & $23.4 \pm 1.8^{*}$ \\
\hline & & $\mathrm{P}$ & $23.7 \pm 2.8$ & $24.1 \pm 2.7$ & $25.9 \pm 2.4$ & $23.9 \pm 2.8$ \\
\hline \multirow[t]{2}{*}{$\mathrm{LDH}\left(\mathrm{U} \mathrm{L}^{-1}\right)$} & \multirow[t]{2}{*}{$115-245$} & $\mathrm{~A}$ & $178.7 \pm 4.8$ & $178.0 \pm 5.0$ & $177.5 \pm 5.3$ & $180.2 \pm 4.8$ \\
\hline & & $\mathrm{P}$ & $181.8 \pm 9.0$ & $174.5 \pm 4.2$ & $174.9 \pm 4.5$ & $180.0 \pm 3.4$ \\
\hline \multirow[t]{2}{*}{$\operatorname{ALP}\left(\mathrm{U} \mathrm{L}^{-1}\right)$} & \multirow[t]{2}{*}{$115-359$} & $\mathrm{~A}$ & $186.3 \pm 6.1$ & $189.4 \pm 8.7$ & $195.3 \pm 7.5$ & $191.9 \pm 6.7$ \\
\hline & & $\mathrm{P}$ & $197.2 \pm 7.5$ & $197.6 \pm 8.0$ & $206.4 \pm 8.3^{*}$ & $206.4 \pm 9.6$ \\
\hline \multirow[t]{4}{*}{$\gamma-\mathrm{GT}\left(\mathrm{U} \mathrm{L}^{-1}\right)$} & \multirow[t]{2}{*}{$\mathrm{M}: \leq 70$} & $\mathrm{~A}$ & $33.8 \pm 4.0$ & $36.5 \pm 5.1$ & $33.1 \pm 3.7$ & $31.6 \pm 3.3$ \\
\hline & & $\mathrm{P}$ & $36.3 \pm 8.0$ & $35.5 \pm 8.2$ & $31.8 \pm 4.2$ & $30.1 \pm 4.4$ \\
\hline & \multirow[t]{2}{*}{$\mathrm{F}: \leq 30$} & $\mathrm{~A}$ & $32.6 \pm 4.5$ & $40.4 \pm 13.4$ & $33.9 \pm 7.1$ & $28.6 \pm 4.4$ \\
\hline & & $\mathrm{P}$ & $28.0 \pm 3.3$ & $26.4 \pm 4.0$ & $34.6 \pm 7.2$ & $31.4 \pm 7.4$ \\
\hline
\end{tabular}

Values are presented as mean \pm standard error; $n=38$ and $n=39$ in the active (A) and placebo (P) groups, respectively. ${ }^{*}$ Significant differences were observed compared with the values at 0 week $(p<0.05)$. No significant differences were observed compared with the placebo group. Abbreviations: ALB, albumin; ALP, alkaline phosphatase; ALT, alanine aminotransferase; AST, aspartate aminotransferase; F, female; $\gamma$-GT, $\gamma$-glutamyl transpeptidase; LDH, lactate dehydrogenase; M, male; T-BIL, total bilirubin; TP, total protein.

Table 8 Changes in blood biochemistry parameters (lipids and glucose)

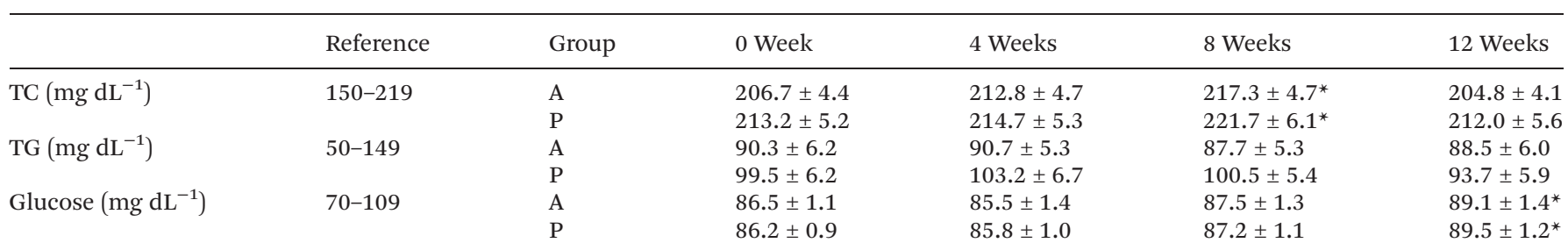

Values are presented as mean \pm standard error. $n=38$ and $n=39$ in the active (A) and placebo (P) groups, respectively. ${ }^{*}$ Significant differences were observed compared with the values at 0 week $(p<0.05)$. No significant difference was observed compared with the placebo group. Abbreviations: TC, total cholesterol; TG, triglyceride.

Table 9 Changes in blood biochemistry parameters (kidney function and electrolytes)

\begin{tabular}{|c|c|c|c|c|c|c|}
\hline & Reference & Group & 0 Week & 4 Weeks & 8 Weeks & 12 Weeks \\
\hline & & $\mathrm{P}$ & $6.01 \pm 0.30$ & $6.05 \pm 0.34$ & $5.80 \pm 0.34$ & $5.97 \pm 0.35$ \\
\hline \multirow[t]{2}{*}{ BUN $\left(\mathrm{mg} \mathrm{dL}^{-1}\right)$} & $8.0-22.0$ & A & $12.59 \pm 0.50$ & $12.11 \pm 0.41$ & $12.15 \pm 0.43$ & $12.77 \pm 0.34$ \\
\hline & & $\mathrm{P}$ & $12.31 \pm 0.39$ & $12.68 \pm 0.43$ & $13.37 \pm 0.46^{*}$ & $12.59 \pm 0.42$ \\
\hline \multirow[t]{2}{*}{$\mathrm{CRE}\left(\mathrm{mg} \mathrm{dL}^{-1}\right)$} & M: 0.61-1.04 & A & $0.816 \pm 0.004$ & $0.822 \pm 0.003$ & $0.830 \pm 0.003$ & $0.809 \pm 0.004$ \\
\hline & & $\mathrm{P}$ & $0.608 \pm 0.001$ & $0.600 \pm 0.001$ & $0.605 \pm 0.001$ & $0.594 \pm 0.001^{*}$ \\
\hline \multirow[t]{2}{*}{$\mathrm{Na}\left(\mathrm{mEq} \mathrm{L} \mathrm{L}^{-1}\right)$} & $136-147$ & A & $140.4 \pm 0.2$ & $139.8 \pm 0.2^{*}$ & $140.0 \pm 0.2$ & $140.2 \pm 0.2$ \\
\hline & & $\mathrm{P}$ & $140.2 \pm 0.2$ & $139.7 \pm 0.2^{*}$ & $139.8 \pm 0.2^{*}$ & $139.8 \pm 0.2^{*}$ \\
\hline \multirow{2}{*}{$\mathrm{Cl}\left(\mathrm{mEq} \mathrm{L}^{-1}\right)$} & 98-109 & A & $105.9 \pm 0.2$ & $104.8 \pm 0.3^{*}$ & $104.8 \pm 0.2^{*}$ & $105.8 \pm 0.3$ \\
\hline & & $\mathrm{P}$ & $106.2 \pm 0.2$ & $105.3 \pm 0.3^{*}$ & $105.3 \pm 0.2^{*}$ & $105.7 \pm 0.2$ \\
\hline \multirow[t]{2}{*}{$\mathrm{K}\left(\mathrm{mEq} \mathrm{L}{ }^{-1}\right)$} & $3.6-5.0$ & A & $4.18 \pm 0.05$ & $4.25 \pm 0.05$ & $4.35 \pm 0.05^{*}$ & $4.29 \pm 0.04^{*}$ \\
\hline & & $\mathrm{P}$ & $4.13 \pm 0.04$ & $4.22 \pm 0.04^{*}$ & $4.27 \pm 0.04^{*}$ & $4.23 \pm 0.04^{*}$ \\
\hline
\end{tabular}

Values are presented as mean \pm standard error; $n=38$ and $n=39$ in the active (A) and placebo (P) groups, respectively. ${ }^{*}$ Significant differences were observed compared with the values at 0 week $(p<0.05)$. No significant differences were observed compared with the placebo group. Abbreviations: BUN, blood urea nitrogen; CRE, creatinine; UA, uric acid. 
for female subjects. In case of Ht values, significant increases were observed at 4 and 8 weeks in the active supplement group for male subjects. The values for $\mathrm{Ht}$ in the active supplement group were significantly lower than those in the placebo group at 0,8 , and 12 weeks for female subjects. In case of PLT count, there were no significant differences between both groups for male and female subjects; however, significant increases were observed at 8 weeks from the value at week 0 in the placebo group for male subjects and in the active supplement group for female subjects. Values of all haematological parameters were within reference ranges.

Urinalysis did not reveal any clinically problematic findings throughout the study. There were significant differences in urine $\mathrm{pH}$ and specific gravity between the two groups after 8 weeks; however, the changes were within reference ranges. There was a significant decrease in urine specific gravity in the active supplement group after 4 and 8 weeks compared to the start value (0 week) (ESI Table $2 \dagger$ ).

During the study period, 17 cases of adverse events were reported in 10 subjects in the placebo group; 9 cases of adverse events were reported in 7 subjects in the active group. In both the groups, cold-like symptoms were the most common adverse events; there was no difference in the number or items of adverse events between the groups. All cases were judged by the site investigators to be of mild to moderate severity, and had no relation to the test supplements.

\section{Discussion}

Obesity increases the risk of metabolic disorders. The global obesity epidemic is rapidly growing; thereby, extensive research is being conducted on drugs and functional foods that can prevent obesity. The Japan Society for the Study of Obesity defines "overweight" as having a BMI of $23-<25 \mathrm{~kg} \mathrm{~m}^{-2}$, while a BMI of $25->30 \mathrm{~kg} \mathrm{~m}^{-2}$ is defined as "obese level 1 (preobese)". ${ }^{24}$

We evaluated the effect of continual intake of polymethoxyflavone purified from KP for 12 weeks, on abdominal visceral fat in Japanese subjects (BMI of 23 or more and less than $30 \mathrm{~kg} \mathrm{~m} \mathrm{~m}^{-2}$ ) without changing their usual lifestyle. Accumulation of visceral fat leads to metabolic syndrome, which is a risk factor for cardiovascular diseases. ${ }^{26}$ Our results show that there was a significant reduction in VFA in the active group compared to the placebo group after 12 weeks.

The subjects were instructed to retain their usual lifestyle habits throughout the study. However, measurement of energy intake and physical activities in this study revealed that in female subjects, calorie intake was significantly lower at 4 weeks of active supplement intake than at 0 week. At 12 weeks, the calorie intake of females in the active intake group was significantly lower than that of females in the placebo group. The effect of transient variations in calorie intake was limited. Further, physical activity levels were significantly decreased in male subjects at 9-12 weeks. Carbohydrate intake was lower in female subjects in the active group compared to the placebo group at 12 weeks, but showed no changes from 0 week. Therefore, the reduction in VFA obtained in the present study may be mainly because of polymethoxyflavone purified from KP. Our results indicate that ingestion of polymethoxyflavone purified from KP reduces VFA in Japanese overweight individuals. Visceral fat is high among smokers. ${ }^{27}$ To assess the effects of smoking, we reconducted the analysis while excluding all smokers, and showed that the changes in VFA at 12 weeks of intake from 0 week was $-3.59 \pm 1.6 \mathrm{~cm}^{2}$ in the active supplement group and $2.33 \pm 1.8 \mathrm{~cm}^{2}$ in the placebo group $(p<0.05)$; this was similar to the results of the analysis wherein all subjects were included (data not shown). It has been suggested that female smokers have a higher visceral fat accumulation than that of male smokers. ${ }^{27}$ In this study, the number of female smokers was small, and may have had little impact on the results.

A previous study has reported a strong correlation between waist to hip ratio and visceral fat. ${ }^{28}$ There was no correlation between waist to hip ratio and VFA in this study. Since this study was conducted on Japanese subjects, the different results obtained were attributed to the mild visceral fat accumulation, contrary to previous reports. ${ }^{28}$

In this study, the body fat ratio was measured using bioelectrical impedance analysis. Body fat percentage was significantly increased at 8 weeks in the active group, and at 4 and 8 weeks in the placebo group. The variation in abdominal fat shown by the CT analysis differed from that of the body fat percentage in this study, possibly because bioelectrical impedance is far less accurate/sensitive than CT.

Results from a previous study showed that ingestion of KPE for 12 weeks reduced VFA, SFA, and TFA in Japanese subjects with a BMI of $24-<30 \mathrm{~kg} \mathrm{~m}{ }^{-2} \cdot{ }^{16}$ We observed a significant reduction in VFA and TFA in the active group, which was administered polymethoxyflavone purified from $\mathrm{KP}$, compared to the placebo group after 8 and 12 weeks. In a previous study in which KPE was administered, ${ }^{16}$ VFA decreased by $3.67 \mathrm{~cm}^{2}$ after 12 weeks of intake, whereas the decrease observed in this study was $3.55 \mathrm{~cm}^{2}$. In both studies, subjects received $12 \mathrm{mg}$ of polymethoxyflavone for 12 weeks; however, the previous study used the extract, and this study used polymethoxyflavone purified from KP. These similar results suggest that the active component of KPE involved in reducing VFA is likely to be polymethoxyflavone.

Since obesity occurs when energy intake exceeds energy expenditure, decrease in energy intake or increase in energy expenditure is important for preventing obesity. ${ }^{29}$ We have previously demonstrated that KPE enhanced energy expenditure in mice by activating brown adipose tissue, and promoting catecholamine secretions in dietary obesity mouse model. ${ }^{20}$ Catecholamines activate hormone-sensitive lipase, and promote lipolysis in adipose tissue. A single intake of KPE increases whole body energy expenditure in humans. ${ }^{22}$ These actions might have contributed to the VFA reduction effect observed in this study. However, it is not known whether polymethoxyflavone purified from KP increases energy expenditure even in the Japanese overweight subjects in this study; the 
actual change in energy expenditure remains undetermined in this study, which is a limitation of this study. The dose of polymethoxyflavone purified from KP used in this study did not induce abnormal physiological changes. The SBP and DBP increased in both groups over the course of 12 weeks. A previous study has shown that SBP and DBP significantly increase during winter than in summer. ${ }^{30}$ This may be attributed to the seasonal variations in outdoor temperatures as well as levels of noradrenaline, catecholamines, and vasopressin. This study was conducted during the summer and winter months of 2019. Therefore, seasonal variations may account for the increase in SBP and DBP in both groups in this study. Similarly, the fasting blood glucose levels increased in both groups over the course of 12 weeks. In a cohort study of diabetes patients from the United States, it was reported that HbA1c showed seasonal variations, with high levels in the winter and low levels in the summer. ${ }^{31}$ The current study was conducted in healthy Japanese subjects, but the intervention period was between September and December; therefore, it is possible that similar seasonal variations in blood glucose levels may have occurred.

Pulse rates in the active supplement group decreased at 8 and 12 weeks. The intake of KPE reportedly reduces stress levels and promotes relaxation, as indicated by heart rate variability analysis. ${ }^{32}$ Therefore, we speculate that polymethoxyflavone purified from KP also reduced pulse rates by regulating autonomic functions.

Moreover, the values of all circulatory parameters, blood biochemistry, haematological parameters, and urinalysis were within the range of physiological variation. However, the $\gamma$-GT levels in the female subjects were slightly higher than the reference range. Further, a temporary increase in $\gamma$-GT levels was also observed in one female subject at 4 weeks in the active group, due to a change in alcohol drinking habit caused by a change in job/career. However, at 8 and 12 weeks, $\gamma$-GT levels returned to their pre-intake levels ( 0 week) with no variation observed in the other subjects. The investigating physician (IF) concluded that there were no changes caused by the intake of polymethoxyflavone purified from KP upon evaluating circulatory parameters (blood pressure and pulse rate), blood biochemistry (liver function, lipids and glucose, and kidney function and electrolytes), haematology, urinalysis, and questioning in this study. The safety of daily consumption of KPE was evaluated in a randomised, double-blind, placebocontrolled trial in a previous report. ${ }^{16}$ Healthy subjects consumed $12 \mathrm{mg}$ polymethoxyflavone for 12 weeks, and no clinically relevant abnormal changes in physical, biochemical, or haematological parameters or in the urinalysis results were reported. ${ }^{16}$ Our group had previously conducted a randomised, double-blind, placebo-controlled clinical study evaluating the safety of daily consumption of $60 \mathrm{mg}$ polymethoxyflavone for 4 weeks. ${ }^{33}$ During the course of the study, no test supplementrelated adverse events or abnormalities in anthropometric, cardiovascular, blood, and urine parameters were observed, when compared to the placebo group. ${ }^{33}$ Moreover, the subchronic toxicity of KPE was investigated in a 90-day oral toxicity study in Sprague-Dawley rats, ${ }^{34}$ while Chivapat et al. evaluated the chronic toxicity of KPE in Wistar rats. ${ }^{35}$ These studies have reported no KPE-associated toxicity. Thus, no adverse events have been reported for KP in previous studies; moreover, no adverse changes caused by polymethoxyflavone purified from KP were observed in this study. Therefore, intake of $12 \mathrm{mg}$ polymethoxyflavone for 12 weeks is considered safe.

A potential limitation of the study was that it did not investigate the long-term effects of the intake of polymethoxyflavone purified from KP. Moreover, additional studies are needed to accurately assess other obesity-related parameters, as this study focused mainly on VFA in healthy subjects. Continuous ingestion of polymethoxyflavone purified from KP for 12 weeks was considered safe; however, to effectively assess its safety, longer periods of intake and higher doses are required to be studied.

In this study, only Japanese adults were included. A metaanalysis by Hursel et al. reported that the body weight reduction effect of green tea extract in Caucasian subjects was weaker than that observed in Asian subjects. ${ }^{36}$ It is possible that the magnitude of visceral fat reduction effect may vary depending on populations. Hence, additional research is required to confirm that polymethoxyflavone purified from KP can exert its visceral fat-lowering effect not only in Japanese but also in other populations.

\section{Conclusions}

This study revealed that regular ingestion of polymethoxyflavone purified from KP reduces visceral fat in Japanese overweight humans, with a BMI of $23 \mathrm{~kg} \mathrm{~m}^{-2}$ or more and less than $30 \mathrm{~kg} \mathrm{~m}^{-2}$, without the need of any lifestyle changes. Intake of polymethoxyflavone purified from KP $(12 \mathrm{mg})$ per day was sufficient to achieve this effect. Therefore, polymethoxyflavone purified from KP could be an effective strategy to prevent visceral fat accumulation.

\section{Conflicts of interest}

Yoshino S, Tagawa T, Awa R, Ogasawara-Mukaida J, and Kuwahara $\mathrm{H}$ are employees of Maruzen Pharmaceuticals Co., Ltd, the study sponsor. Fukuhara I was the site investigator.

\section{Acknowledgements}

We thank all subjects who participated in this study, the staff of medical scientists from Mediffom Inc. and the New Drug Research Center, Inc., and Dr Sato from the New Drug Research Center for performing statistical analysis using a mixed model. We also thank the group members of Maruzen Pharmaceuticals Co., Ltd for their technical support and valuable guidance. We would like to thank Editage for English language editing. 


\section{References}

1 P. Mathieu, P. Pibarot and J. P. Despres, Metabolic syndrome: the danger signal in atherosclerosis, Vasc. Health Risk Manage, 2006, 2, 285-302, DOI: 10.2147/ vhrm.2006.2.3.285.

2 B. B. Kahn and J. S. Flier, Obesity and insulin resistance, J. Clin. Invest., 2000, 106, 473-481, DOI: 10.1172/JCI10842.

3 R. H. Eckel, S. M. Grundy and P. Z. Zimmet, The metabolic syndrome, Lancet, 2005, 365, 1415-1428, DOI: 10.1016/ S0140-6736(05)66378-7.

4 I. R. Hsu, S. P. Kim, M. Kabir and R. N. Bergman, Metabolic syndrome, hyperinsulinemia, and cancer, Am. J. Clin. Nutr., 2007, 86, s867-s871, DOI: 10.1093/ajcn/ 86.3.867S.

5 K. N. Frayn, Visceral fat and insulin resistance - causative or correlative?, Br. J. Nutr., 2000, 83, S71-S77, DOI: 10.1017/ s0007114500000982.

6 H. Tsuji, M. Kasai, H. Takeuchi, M. Nakamura, M. Okazaki and K. Kondo, Dietary medium-chain triacylglycerols suppress accumulation of body fat in a double blind, controlled trial in healthy men and women, J. Nutr., 2001, 131, 2853-2859, DOI: 10.1093/jn/131.11.2853.

7 R. Hursel, W. Viechtbauer, A. G. Dulloo, A. Tremblay, L. Tappy, W. Rumpler and M. S. Westerterp-Plantenga, The effects of catechin rich teas and caffeine on energy expenditure and fat oxidation: a meta-analysis, Obes. Rev., 2011, 12, 573-581, DOI: 10.1111/j.1467-789X.2011.00862x.

8 Y. Morimoto-Kobayashi, K. Ohara, H. Ashigai, T. Kanaya, K. Koizumi, F. Manabe, Y. Kaneko, Y. Taniguchi, M. Katayama, Y. Kowatari and S. Kondo, Matured hop extract reduces body fat in healthy overweight humans: a randomized, double-blind, placebo-controlled parallel group study, Nutr. J., 2016, 15, 25, DOI: 10.1186/s12937016-0144-2.

9 L. Trigueros, S. Pena, A. V. Ugidos, E. Sayas-Barberá, J. A. Pérez-Álvarez and E. Sendra, Food ingredients as antiobesity agents: a review, Crit. Rev. Food Sci. Nutr., 2013, 53, 929-942, DOI: 10.1080/10408398.2011.574215.

10 M. Fukushima, H. Suzuki and Y. Seino, Insulin secretion capacity in the development from normal glucose tolerance to type 2 diabetes, Diabetes Res. Clin. Pract., 2004, 66, S37S43, DOI: 10.1016/j.diabres.2003.11.024.

11 M. Ryo, K. Kishida, T. Nakamura, T. Yoshizumi, T. Funabashi and I. Shimomura, Clinical significance of visceral adiposity assessed by assessed by computed tomography: A Japanese perspective, World J. Radiol., 2014, 6, 409-416, DOI: 10.4329/wjr.v6.i7.409.

12 T. Akase, T. Shimada, S. Terabayashi, Y. Ikeya, H. Sanada and M. Aburada, Antiobesity effects of Kaempferia parviflora spontaneously obese type 2 diabetic mice, J. Nat. Med., 2011, 65, 73-80, DOI: 10.1007/s11418-010-0461-2.

13 D. Chen, H. Li, W. Li, S. Feng and D. Deng, Kaempferia parviflora and its methoxyflavones: chemistry and biological activities, Evid. Based. Complement. Alternat. Med, 2018, ID4057456, DOI: 10.1155/2018/4057456.
14 K. Sutthanut, B. Sripanidkulchai, C. Yenjai and M. Jay, Simultaneous identification and quantitation of 11 flavonoid constituents in Kaempferia parviflora by gas chromatography, J. Chromatogr. A, 2007, 1143, 227-233, DOI: 10.1016/j.chroma.2007.01.033.

15 D. Bei and G. An, Pharmacokinetics and tissue distribution of 5,7-dimethoxyflavone in mice following single dose oral administration, J. Pharm. Biomed. Anal., 2016, 119, 65-70, DOI: 10.1016/j.jpba.2015.11.025.

16 S. Yoshino, R. Awa, Y. Miyake, I. Fukuhara, H. Sato, T. Ashino, S. Tomita and H. Kuwahara, Daily intake of Kaempferia parviflora extract decreases abdominal fat in overweight and preobese subjects: a randomized, doubleblind, placebo-controlled clinical study, Diabetes, Metab. Syndr. Obes.: Targets Ther., 2018, 11, 447-458, DOI: 10.2147/DMSO.S169925.

17 C. Sae-wong, P. Tansakul and S. Tewtrakul, Anti-inflammatory mechanism of Kaempferia parviflora in murine macrophage cells (RAW 264.7) and in experimental animals, J. Ethnophamacol., 2009, 124, 576-580, DOI: 10.1016/j. jep.2009.04.059.

18 S. K. Wattanapitayakul, M. Suwatronnakorn, L. Chularojmontri, A. Herunsalee, S. Niumsakul, S. Charuchongkolwongse and N. Chansuvanich, Kaempferia parviflora ethanolic extract promoted nitric oxide production in human umbilical vein endothelial cells, J. Ethnopharmacol., 2007, 110, 559-562, DOI: 10.1016/j.jep.2006.09.037.

19 W. Kusirisin, S. Srichairatanakool, P. Lerttrakarnnon, N. Lailerd, M. Suttajit, C. Jaikang and C. Chaiyasut, Antioxidative activity, polyphenolic content and anti-glycation effect of some Thai medicinal plants traditionally used in diabetic patients, Med. Chem., 2009, 5, 139-147, DOI: 10.2174/157340609787582918.

20 S. Yoshino, M. Kim, R. Awa, H. Kuwahara, Y. Kano and T. Kawada, Kaempferia parviflora extract increases energy consumption through activation of BAT in mice, Food Sci. Nutr., 2014, 2, 634-637, DOI: 10.1002/fsn3.144.

21 T. Shimada, T. Horikawa, Y. Ikeya, H. Matsuo, K. Kinoshita, T. Taguchi, K. Ichinose, K. Takahashi and M. Aburada, Preventive effect of Kaempferia parviflora ethyl acetate extract and its major components polymethoxyflavonoid on metabolic diseases, Fitoterapia, 2011, 82, 1272-1278, DOI: 10.1016/j.fitote.2011.08.018.

22 M. Matsushita, T. Yoneshiro, S. Aita, T. Kamiya, N. Kusaba, K. Yamaguchi, K. Takagaki, T. Kameya, H. Sugie and M. Saito, Kaempferia parviflora extract increases whole-body energy expenditure in humans: roles of brown adipose tissue, J. Nutr. Sci. Vitaminol., 2015, 61, 79-83, DOI: 10.3177/jnsv.61.79.

23 S. Yoshino, R. Awa, Y. Miyake, H. Kuwahara, Y. Akamatsu and T. Moritani, Effects of single oral intake of Kaempferia parviflora extract on energy metabolism-a randomized double-blind crossover study, Jpn. Pharmacol. Ther., 2016, 44, 1757-1762.

24 The Examination Committee of Criteria for 'Obesity Disease' in Japan, Japan Society for the Study of Obesity, 
Japan Society for the Study of Obesity, New Criteria for 'Obesity Disease' in Japan, Circ. J., 2002, 66, 987-992.

25 K. Tokunaga, Y. Matsuzawa, K. Ishikawa and S. Tarui, A novel technique for the determination of body fat by computed tomography, Int. J. Obes., 1983, 7, 473-445.

26 J. P. Despres, Body fat distribution and risk of cardiovascular disease: an update, Circulation, 2012, 126, 13011313, DOI: 10.1161/CIRCULATIONAHA.111.067264.

27 K. Nakanishi, M. Nishida, T. Ohama, T. Moeiyama and K. Yamauchi-Takihara, Smoking associates with visceral fat accumulation especially in women, Circulation J., 2014, 78, 1259-1263, DOI: 10.1253/circj.cj-13-1134.

28 F. F. Ribeiro-Filho, A. N. Faria, S. Azjen, M. T. Zanella and S. R. Ferreira, Methods of estimation of visceral fat: advantages of ultrasonography, Obes. Res., 2003, 11, 1488-1494, DOI: 10.1038/oby.2003.199.

29 B. M. Spiegelman and J. S. Flier, Obesity and the regulation of energy balance, Cell, 2001, 104, 531-543, DOI: 10.1016/ s0092-8674(01)00240-9.

30 T. Iwahori, K. Miura, K. Obayashi, T. Ohkubo, H. Nakajima, T. Shiga and H. Ueshima, Seasonal variation in home blood pressure: findings from nationwide web-based monitoring in Japan, BMJ Open, 2018, 8, e017351, DOI: 10.1136/ bmjopen-2017-017351.

31 C. L. Tseng, M. Brimacombe, M. Xie, M. Rajan, H. Wang, J. Kolassa, S. Crystal, T. C. Chen, L. Pogach and M. Safford,
Seasonal patterns in monthly hemoglobin A1c values, Am. J. Epidemiol., 2005, 161, 565-574, DOI: 10.1093/aje/ kwi071.

32 W. Eungpinichpong, U. Chatchawan, B. Sripanidkulchai, S. Arunpongpaisal and W. Chompoopan, Effects of Kaempferia parviflora on physical and psychological stresses in adults, Int. J. GEOMATE, 2018, 15, 26-31, DOI: 10.21660/ 2018.50.3665.

33 S. Yoshino, R. Awa, Y. Miyake, I. Fukuhara, H. Sato, Y. Endo, S. Tomita and H. Kuwahara, Evaluation of the safety of daily consumption of Kaempferia parviflora extract (KPFORCE): a randomized double-blind placebo-controlled trial, J. Med. Food, 2019, 22, 1168-1174, DOI: 10.1089/ jmf.2018.4372.

34 S. Yoshino, R. Awa, N. Ohto, Y. Miyake and H. Kuwahara, Toxicological evaluation of standardized Kaempferia parviflora extract: sub-chronic and mutagenicity studies, Toxicol. Rep., 2019, 6, 544-549, DOI: 10.1016/j.toxrep.2019.06.003.

35 S. Chivapat, P. Chavalittumrong, A. Attawish and A. Rungsipipat, Chronic toxicity study of Kaempferia parviflora Wall ex. extract, Thai J. Vet. Med., 2010, 40, 377383.

36 R. Hursel, W. Viechtbauer and M. S. Westertero-Plantenga, The effects of green tea on weight loss and weight maintenance: a meta-analysis, Int. J. Obes., 2009, 33, 956-961, DOI: 10.1038/ijo.2009.135. 